\title{
Plant Pectin: A Potential Source for Cancer Suppression
}

\author{
Suryakant K. Niture and Lubna Refai \\ Department of Pharmacology and Experimental Therapeutics, \\ University of Maryland School of Medicine, 655 West Baltimore Street, Baltimore, MD 21201, United States
}

Received 2013-01-04, Revised 2013-01-31; Accepted 2013-04-18

\begin{abstract}
Pectin is a branched polysaccharide present widely in a variety of fruits. It has been of high interest to cancer biologists due to its involvement in metal/carcinogen detoxification and its anti-carcinogenic properties. In particular, Citrus Pectin (CP) and Modified Citrus Pectin (MCP) have been shown to have a significant inhibitory role in cancer cell metastasis, invasion, angiogenesis and survival. The interactions and inactivation of oncogenes by $\mathrm{CP}$ and $\mathrm{MCP}$ in prostate, breast, liver, lung, melanoma and multiple myeloma cancers suggest that $\mathrm{CP}$ and MCP could play an important role in cancer chemotherapy and chemoprevention. In this review, we focus on the biological function of pectin with respect to its properties, sources, structure and its potential role in carcinogen detoxification and cancer suppression.
\end{abstract}

Keywords: Citrus Pectin, Modified Citrus Pectin, Cancer Prevention

\section{INTRODUCTION}

In today's world, particularly in developing countries, humans are exposed more than ever to a wide variety of toxins. Toxins are present in every environment such as the work place and home and can be found in the air, food and water (Brunekreef and Holgate, 2002; Perez-Padilla et al., 2010). Interestingly, we also generate toxins within our own body due to the improper metabolism of our diet. Toxin accumulation in the body can occur by exposure to a toxic environment or by consuming an improper diet (Jew et al., 2009). This can cause pain, irritation and inflammation in the body. Toxins impose a large burden on our digestive system which leads to a decreased detoxification process. Acute/short exposure to toxins in cells and tissues produce free radicals which damage organ function over time. Long term toxin exposure may result in metabolic and genetic alterations that can affect cell growth and immune response. This is due to the interaction of toxins/carcinogens with bio-molecules such as DNA and proteins (Jackson and Bartek, 2009). Many times environmental toxins also act as a potent carcinogen (Sankpal et al., 2012). Data from the World Health Organization (WHO) suggests that environmental toxins, as well as other factors, caused 7.6 million cancer deaths in 2008 and is expected to increase to 11.4 million by 2030 (Jemal et al., 2010). Once these toxic chemicals/metals accumulate in the body, they cannot be fully eliminated unless they undergo a detoxification process. If detoxification fails to eliminate these toxins, they may cause the development of severe diseases such as cancer, lung diseases, diabetes, chronic fatigue and arthritis (Landrigan et al., 2002; Boyd and Genuis, 2008). A healthy diet is an essential tool to reduce and avoid the accumulation of toxic substances in our body.

Several plant products contain carbohydrates which possess great medicinal values. Carbohydrates have enormous potential not only in producing energy, but also in regulating biological processes. All human cells express carbohydrates on their surfaces in the form of glycoproteins, glycolipids and polysaccharides. Emerging studies indicate that plant pectin, a complex branched polysaccharide fiber rich in galactoside

Corresponding Address: Suryakant K. Niture, Department of Pharmacology and Experimental Therapeutics, University of Maryland School of Medicine, 655 West Baltimore Street, Baltimore, Maryland 21201, United States Tel: 410 706-2284 
residues, has been shown to play critical therapeutic roles against various cancers (Olano-Martin et al., 2003; Glinsky and Raz, 2009). Reports also suggest that pectic polysaccharides are involved in an immuno-modulation process (Wong et al., 1994) and possesses anti-ulcer effects (Kiyohara et al., 1994). Due to its complex structure, pectin has limited solubility in water and, therefore, the bio-arability of pectin in the human body is significantly low. Unfortunately, humans cells are unable to produce pectin degrading enzymes to degrade the pectin; therefore, scientists have developed Modified forms of Citrus Pectin (MCP) (Baldwin and Shah, 1993; Pienta et al., 1995). Several recent independent studies have shown that MCP plays an important role in carcinogen detoxification and cancer suppression by its interaction with different oncogenes in cancer cells (Glinsky and Raz, 2009). MCP has been shown to play a more potential therapeutic role against various cancers compared to normal citrus pectin (Hayashi et al., 2000). Fifty independent studies have demonstrated that MCP interacts with galectin-3 and inhibits tumor growth or cancer metastasis in vivo and in vitro in various types of cancers including prostate, colon, breast, melanoma and multiple myeloma (Pienta et al., 1995; Hsieh and $\mathrm{Wu}$, 1995; Glinskii et al., 2005; Hayashi et al., 2000; NangiaMakker et al., 2002; Sathisha et al., 2007; Inohara and Raz, 1994; Glinsky and Raz, 2009). In this review, we focus on pectin and modified citrus pectin, their structural properties and their important role in detoxification and cancer suppression.

\subsection{Pectin Structure and Some Properties}

Pectin occurs as a constituent of higher-plant cell walls embedded in the cellulose fibrils. Pectin molecules are an extremely complex and structurally diverse group of polymers. The fine structures of pectins can be widely heterogeneous between plants, tissues and even within a single cell wall (Willats et al., 2001). The composition of pectin varies in plant species and is dependent on the age and maturity of the plant parts. Although researchers have reported several possible structures of pectin molecules, it is extremely difficult to predict its fine exact structure. The complex structure of pectin polysaccharide contains 1,4 -linked $\alpha$-D-galactosyluronic acid residues. Three pectic polysaccharides have been isolated from plant primary cell walls and structurally characterized (Fig. 1). These are: homogalacturonans, substituted galacturonans and rhamnogalacturonans. Homogalacturonans are linear chains of $\alpha-(1-4)$-linked D-galacturonic acid. Substituted galacturonans are characterized by the presence of saccharide appendant residues (such as D-xylose or D-apiose in the respective cases of xylogalacturonan and apiogalacturonan) branching from a backbone of D-galacturonic acid residues. Rhamnogalacturonan I pectins (RG-I) contain a backbone of the repeating disaccharide: $(1,4)-\alpha-\mathrm{D}$ galacturonic acid-(1,2)- $\alpha$-L-rhamnose-(1) (Ridley et al., 2001; Vincken et al., 2003). From many of the rhamnose residues, side chains of various neutral sugars branch off (Fig. 1). The neutral sugars are mainly D-galactose, Larabinose and D-xylose with the types and proportions of neutral sugars varying with the origin of pectin. Another structural type of pectin is Rhamnogalacturonan II (RGII), a less frequent complex, which is a highly branched polysaccharide. Rhamnogalacturonan II is classified by some authors within the group of substituted galacturonans since the rhamnogalacturonan II backbone is made exclusively of D-galacturonic acid units (Vincken et al., 2003; Niture, 2008). Isolated pectin has a molecular weight of typically $60-130,000 \mathrm{~g} / \mathrm{mol}$ and varies in origin and extraction conditions. In nature, around $80 \%$ of carboxyl groups of galacturonic acid are esterified with methanol and this proportion is decreased, more or less, during pectin extraction. The behavior of pectin in food applications is determined by the ratio of esterified to non-esterified galacturonic acid. Galacturonic acids of homogalacturonan may be both methyl-esterified and acetylated. Both the degree of methyl-esterification and degree of acetylation have an impact on functional properties of pectin. When esterification is more than $50 \%$, the homogalacturonan is termed as high-methoxyl pectin, whereas when the degree of esterification is less than $50 \%$ it is termed as lowmethoxyl pectin (Voragen et al., 2010; Rinaldo, 1996). The methylesterification of homogalacturonan, in particular, has drawn the attention of many research groups because it determines the industrial applicability of pectin. Not only is the amount of methyl-esterification important, but also the distribution of methyl groups on the homogalacturonan backbone. Blocks of more than 10 unesterified galacturonic acid residues generally yield a pectin molecule, which is sensitive to $\mathrm{Ca} 2+$ cross-linking (Daas et al., 2001). Various physical and chemical factors such as temperature, $\mathrm{pH}$, degree of methylation, attachment of different sugars, calcium ion and other solutes affect the gelling properties as well as pectin hydrolysis (Krall and Mcfeeters, 1998). Generally, homogalacturonan regions of pectin are termed as 'smooth-regions' and rhamnogalacturonan regions as 'hairy/branched regions'. The non-esterified galacturonic acid units can be either free acids (carboxyl groups) or salts with sodium, potassium or calcium. 


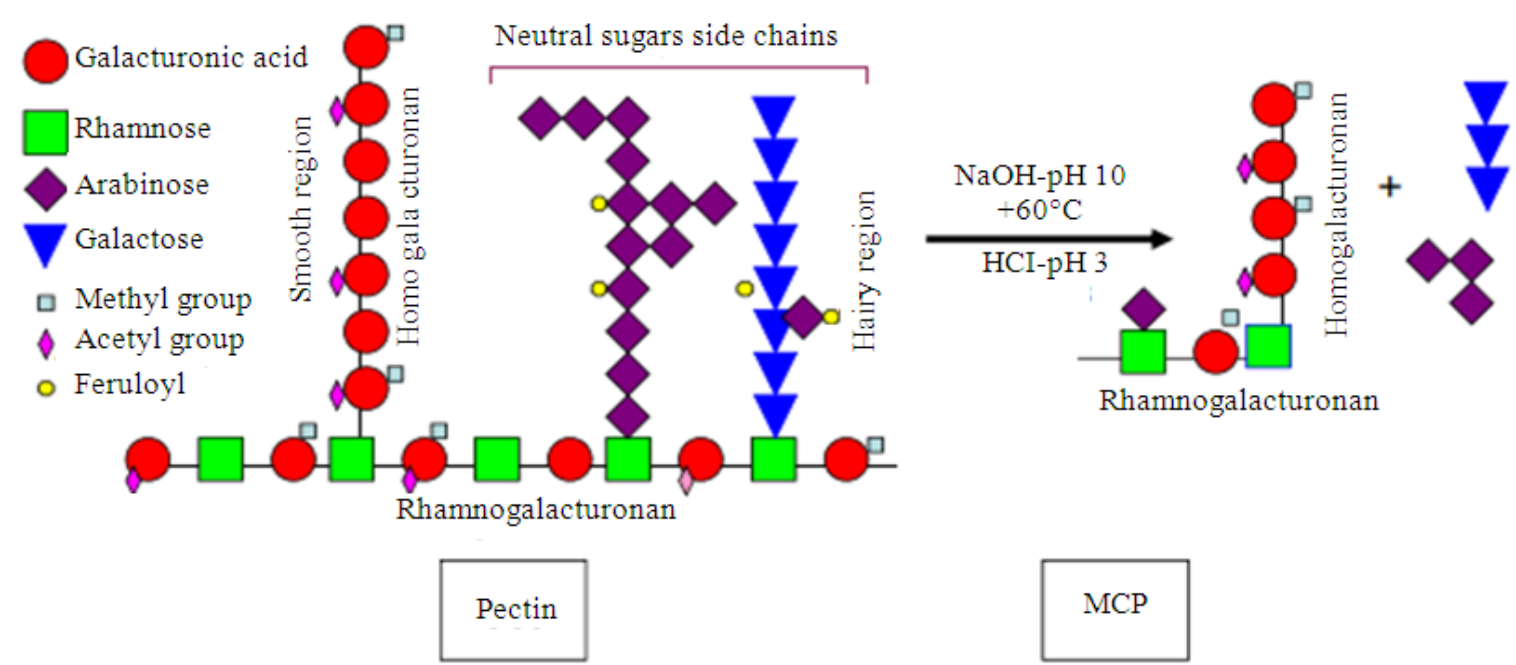

Fig. 1. Diagrammatic representation of different components of pectin molecule. The associated neutral sugars side-chains also presented (Vincken et al., 2003). Diagram also represents preparation of MCP from pectin (Pienta et al., 1995).

The salts of partially esterified pectins are called pectinates. If the degree of esterification is below $5 \%$ the salts are called pectates and the insoluble acid forms are pectic acids (Krall and Mcfeeters, 1998). Sugar beet, potato and pear pectins are acetylated at galacturonic acid and methyl esters. Acetylation prevents gelformation but increases the stabilizing and emulsifying effects of pectin.

\subsection{Sources and Extraction of Pectin}

Pectin is abundantly present in citrus fruits such as oranges, lemons and grapefruits. Fruits like apples, guavas, quince, plums and gooseberries contain high levels of pectin, while soft fruits like cherries, grapes and strawberries contain lower amounts (Srivastava and Malviya, 2011). The levels of pectin found in plants are (fresh weight): apples, $1-1.5 \%$; apricot, $1 \%$; cherries, $0.4 \%$; oranges $0.5-3.5 \%$; carrots $1.4 \%$; citrus peels, $30 \%$. The main raw-materials for pectin production are dried citrus peel or apple pomace, which are both by-products of juice production. Pomace from sugar-beets is also used to a small extent. From these materials, pectin is extracted by adding hot dilute mineral acid near a $\mathrm{pH}$ of 2.0 (Joye and Luzio, 2000). During several hours of extraction, the protopectin loses some of its branching and chain-length and goes into solution. After filtering, the extract is concentrated in a vacuum and the pectin is then precipitated by adding either ethanol or isopropanol. Alcohol-precipitated pectin is then separated, washed and dried. Treating the initial pectin with dilute acid leads to low-esterified pectins (Joye and Luzio, 2000).
When this process includes ammonium hydroxide, amidated pectins are obtained. After drying and milling pectin is usually standardized with sugar, calcium-salts or organic acids to have optimum performance in a particular application. Suitable pectins can therefore be selected for the preparation of jams, jellies, or for higher sugar confectionery jellies (May, 1990). Today, several plant pectins are also used in the health care system due to its potent medicinal values.

\subsection{Preparation Modified Citrus Pectin (MCP)}

Plant pectins are highly branched complex molecules and, therefore, their solubility is low. This fact creates a large obstacle in regards to the application of pectin in the medicinal field. Scientists have developed Modified Citrus Pectin (MCP), which increases the ability of pectin to biologically interact with biomolecules such as proteins and lipids. Baldwin and Shah (1993) and Pienta et al. (1995) have prepared modified citrus pectin by treating the pectin molecules with different $\mathrm{pH}$ levels and temperatures. First, citrus pectin is briefly solubilized as a $1.5 \%$ solution in distilled water and its $\mathrm{pH}$ is increased to 10.0 with $\mathrm{NaOH}(3 \mathrm{~N})$ for $1 \mathrm{~h}$ at $50-60^{\circ} \mathrm{C}$. The solution is then cooled to room temperature, while its $\mathrm{pH}$ was adjusted to 3.0 with $3 \mathrm{~N}$ $\mathrm{HCl}$ and stored overnight. Samples are precipitated the next day with $95 \%$ ethanol and incubated at $-20^{\circ} \mathrm{C}$ for 2 h. Lastly, the samples are filtered, washed with acetone and dried on Whatman filters (Fig. 1).

Generally, MCP has a molecular weight of 15400 $\mathrm{g} / \mathrm{mol}$ and is a mostly linear homogalacturonan with a 
$3.8 \%$ esterification and about $10 \%$ rhamnogalacturonan II (Eliaz et al., 2006). MCP can be used for experimental purposes or given to patients. MCP is more easily absorbed by the digestive tract than normal citrus pectin and is then absorbed into the bloodstream. MCP is available as a powder or a capsule. The dose suggested by manufacturers for the powder is five grams (nearly a fifth of an ounce) mixed with water or juice and taken three times a day with meals (Nicholas, 2009). As suggested by American Cancer Society (http://www.cancer.org/) the suggested dose for capsules (pecta-Sol) is 800 milligrams three times a day with meals.

\subsection{Pectin and Detoxification}

Humans are unable to degrade pectin molecules due to a lack of secretion of pectic enzymes; however, the consumption of pectin can potentially have an effect in the detoxification of hazardous chemicals and metals from the human body. Studies have also shown that pectin plays an important role in digestive tract cleaning. Consumption of pectin not only reduces metal toxicity from the body but also helps in reducing metal related inflammation, reducing cholesterol levels, removing artery plaque, eliminating arthritic pain and stiffness, eliminating constipation and diarrhea and regulating blood glucose levels (Jenkins et al., 1975: 1977; Fisher et al., 1966). Several recent clinical studies (Rabbani et al., 2001; Triplehorn and Millard, 2002) have demonstrated that oral pectin supplementation to children and infants reduce acute intestinal infections and significantly slow diarrhea. It is thought that this is due to the reduction of pathogenic bacteria such as Shigella, Salmonella, Klebsiella, Enterobacter, Proteus and Citrobacter from intestine. This is supported by Olano-Martin et al. (2002) who observed that pectin stimulated the growth of certain strains of Bifidobacteria and Lactobacillus in vitro. These bacteria are considered to be directly related to the health of the large intestine and their concentrations depict a healthy microflora population. Pectin also seems to be connected with heart health. The quality of fibrin is thought to be an important risk factor for atherosclerosis, stroke and coronary heart disease. Veldman's work found that pectin managed to increase fibrin permeability and decrease fibrin tensile strength in hyperlipidaemic men (Veldman et al., 1999). Although the mechanism behind this is unknown, it is thought to be due in part to acetate production. Pectin predominantly yields acetate in the colon which is thought to enter peripheral circulation and alter fibrin architecture.
The second important aspect about pectin is its use in chelation therapy. The standard western medical approach for removing heavy metals from the body is chelation therapy. This procedure, performed with harsh chelators introduced intravenously, can cause multiple side effects while potentially robbing the body of some of its essential nutrients. Citrus pectin, however, works as a gentle chelator in the bloodstream and is very useful for ongoing detoxification with little side effects. Two recent clinical studies have found that citrus pectin is a very safe and effective supplement for reducing the heavy metal load in the body. A clinical study was conducted recently where citrus pectin was administered to a group of volunteers and their baseline levels of total body mercury burden was compared before and after four months of citrus pectin treatment (Eliaz et al., 2007). The results showed a significant average decrease (over 60\%) in the total body mercury burden after treatment with citrus pectin. In other studies, patients who had been given citrus pectin were proven to increase urinary secretion of heavy metals such as lead, mercury, cadmium and arsenic (Eliaz et al., 2006; Zhao et al., 2008). All these studies suggest that citrus pectin is a viable alternative to the harsher intravenous chelating therapies as citrus pectin was found to be both effective and free of any side effects.

\subsection{Pectin, MCP and Cancer}

The American Cancer Society suggests that adults eat five servings of fruits and vegetables each day in order to prevent cancer risk indicating the potential and essential fruit pectin role in cancer prevention (Kushi et al., 2006). Due to poor solubility, pectin provides small nutrient content; however, pectin provides an important source of dietary fiber. Several independent research studies have shown that pectin not only benefits health-promoting properties but also reduces cancer risk. Citrus pectin and modified citrus pectin is useful in the prevention and treatment of metastatic cancer, especially in solid tumors like melanoma and cancers of the prostate, colon and breast (Nicholas, 2009). In addition, MCP inhibits cancer progression by suppressing angiogenesis and metastasis of various cancer types in animal models (Glinskii et al., 2005; Glinsky and Raz, 2009; Johnson et al., 2007). The possible role of MCP in the suppression of metastasis is presented in Fig. 2. MCP is rich in galactose residues, which are easily processed by the digestive system and absorbed into the bloodstream (Nicholas, 2009). 


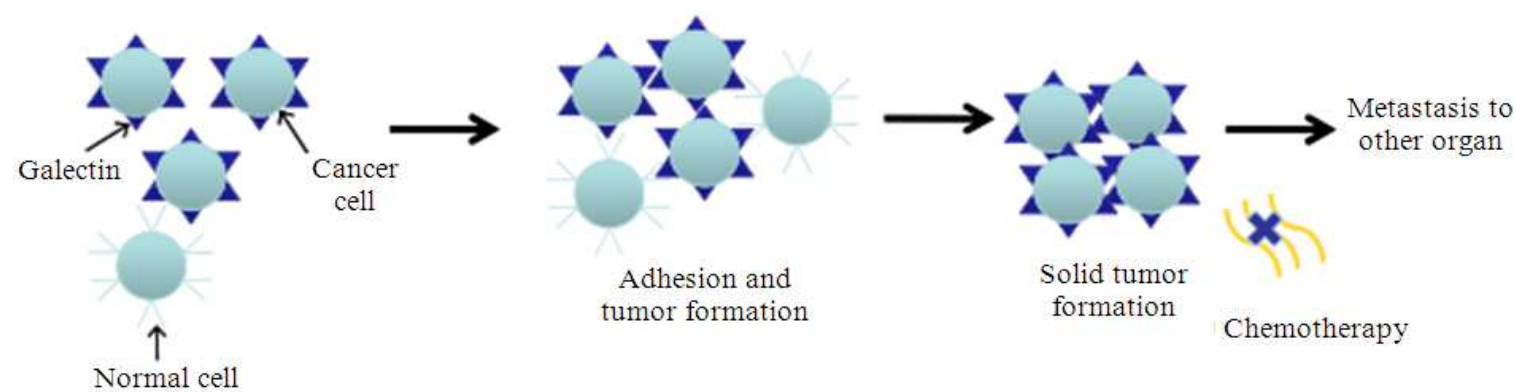

(A)

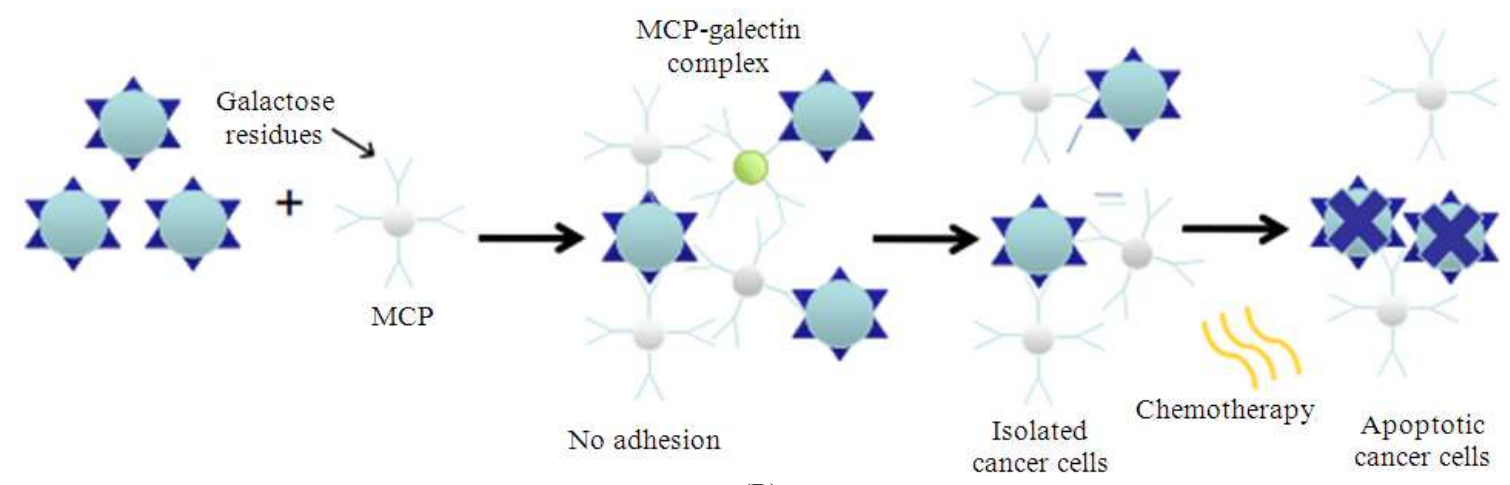

(B)

Fig. 2. (A) A possible mechanism of cancer metastasis is presented. (B) The inhibitory role of MCP-galectin complex interaction in cancer cell metastasis is presented

Scientists continuously give more attention to $\mathrm{MCP}$ because it possesses more active and effective biological properties. Studies have been conducted and show that MCP effectively reduces the metastasis of prostate cancer. Pienta et al. (1995) were the first to show that MCP reduced prostate cancer metastasis in rats. In their investigation, rats were injected with human prostate cancer cells and divided into four groups. The control group received only water and the other groups received water with different concentrations of MCP. After one month, the metastasis of prostate cancer cells into lung tissue was analyzed. The results showed only $50 \%$ of the rats that drank water with MCP $(0.1 \%$ weight/volume) had any metastases, while $94 \%$ of the rats that drank plain water had cancer metastasize to their lungs. Similarly, several animal studies have found that MCP helps reduce the spread of not only prostate cancer but also breast, skin and liver cancer (Nangia-Makker et al., 2002; Platt and Raz, 1992; Liu et al., 2008). MCP was fed to mice with these types of cancer and the mice were found to have a much lower chance of the tumor spreading to the lungs. In another animal study, the effects of MCP on lung metastasis from melanoma cells were examined
(Platt and Raz, 1992). Mice injected with melanoma cells and given MCP were shown to have significantly fewer tumors spread to the lungs compared with mice that did not receive the MCP. When lung tumors did develop in the mice treated with MCP, the tumors tended to be smaller than those that formed in the untreated mice (Platt and Raz, 1992). In one human study, it was shown that MCP increases the Prostate Specific Antigen (PSA) doubling time (Guess et al., 2003). In this phase II pilot study of ten men whose prostate cancer had returned after an initial treatment with surgery or radiation, Prostate Specific Antigen Doubling Time (PSADT) increased in eight $(80 \%)$ of the ten men after taking MCP for twelve months. These studies appear to show that the presence of MCP makes it difficult for cancer cells to break off from the main tumor, aggregate and spread to other organs.

\subsection{Pectin, MCP and Cancer Cell Apoptosis}

Although CP or MCP is not capable to prevent $100 \%$ metastatic tumor formation, recent reports suggest that pectic polymer, $\mathrm{CP}$ or $\mathrm{MCP}$, induces cellular apoptosis in various cancers. 


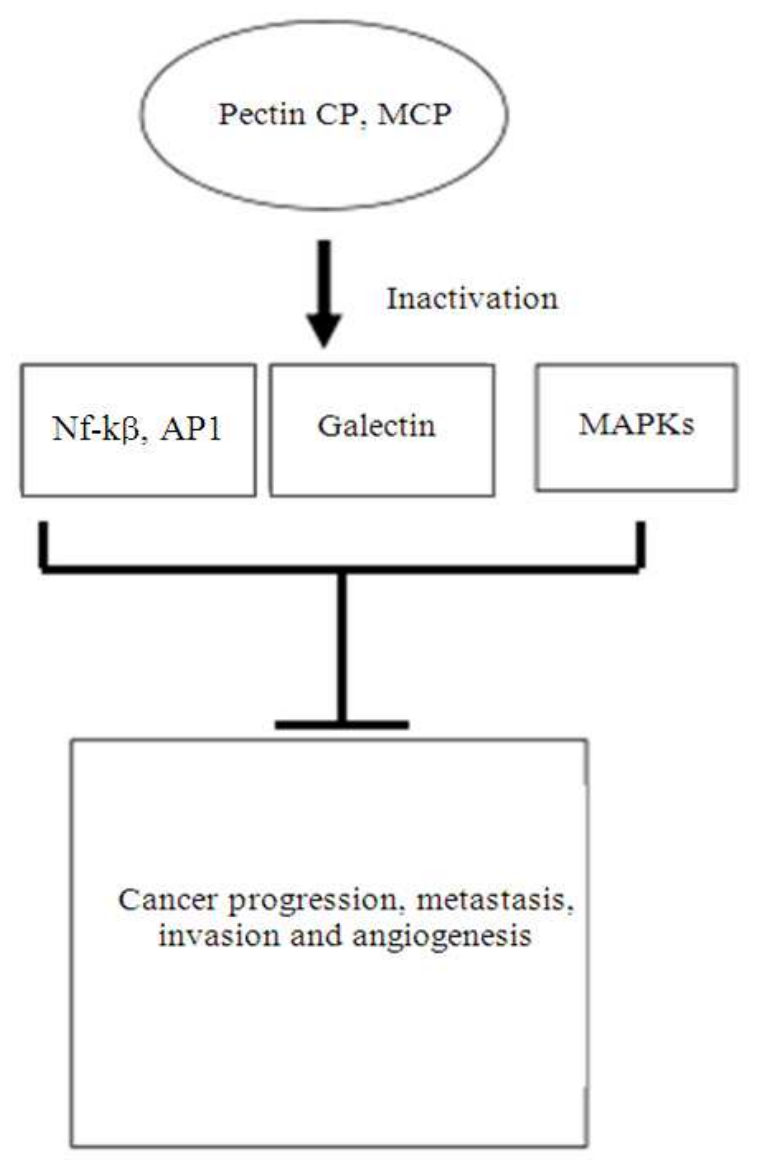

Fig. 3. The sketch diagram represents the molecular intervention between pectin, $\mathrm{CP}$ and $\mathrm{MCP}$ with different factors in cancer cell

Jackson et al. (2007) have shown that commercially available plant pectin is effectively capable to induce apoptosis in androgen responsive LNCap and androgen independent LNcaP-C4-2 prostate cancer cells compared with $\mathrm{CP}, \mathrm{pH}$ modified $\mathrm{CP}$ or pectaSol suggesting that specific structural elements within pectin are responsible for the apoptotic activity. In the case of colon cancer cells, Cheng et al. (2011) have demonstrated that treatment of HT-29 human colon cancer cells with temperature modified HG-rich pectin dramatically increased anti-proliferative effect in the cells. The data also suggests that treatment of HG-rich pectin induces $\mathrm{G} 2 / \mathrm{M}$ cell cycle arrest, activates caspase3 and promotes apoptosis. Similarly, results were reported earlier that pectin and pectic-oligosaccharides also induce DNA fragmentation which leads to cellular apoptosis in HT29 colon cancer cells (Olano-Martin et al., 2003). Yan and Katz (2010) showed that treatment of
PectaSol-C modified citrus pectin (1\%) inhibits MAP kinase activation, cell proliferation/cell survival and induces apoptosis in human and mouse androgendependent and -independent prostate cancer cells. Recently, Ramachandran et al. (2011) showed that MCP activates of human blood lymphocyte subsets like T, B and NK-cells. The data also suggests that MCP activates NK-cells and induces cell death in K562 leukemia cancer cells suggesting that unsaturated oligogalacturonic acids appear to be immunostimulatory. These evidences clearly suggest that MCP holds significant anticarcinogenic potential, particularly, by up-regulation cancer cell apoptosis.

\subsection{Pectin, MCP and their Molecular Interaction in Cancer}

The molecular mechanism of how pectin and MCP suppress cancer metastasis/progression was investigated in limited studies. The research data from various investigations suggest that MCP interacts with galectin3, a 31-42 $\mathrm{kDa}$ protein. Galectin-3 belongs to the galectin super-family of proteins, defined by a shared conserved sequence of the carbohydrate-binding domain and possesses high affinity towards $\beta$-galactosides (Sathisha et al., 2007). Galectin-3 is present in abnormally high levels in many cancers and plays an important role in the growth, survival and spread of cancer cells (Bresalier et al., 1997; Johnson et al., 2007). Galectin-3 is highly expressed on tumor cell surfaces and a variety of metastatic cancer cells and is closely related to cell to cell adhesion, aggregation of cancer cells in vitro, tumor growth and metastasis in vivo (Bresalier et al., 1997; Dumic et al., 2006; Krzeslak and Lipinska, 2004). A notable feature of galectin-3 is its implication in neoplastic transformation and cancer progression. A direct relationship has been shown between galectin-3 levels and the stage of progression of some tumors (Inohara and Raz, 1994; Willats et al., 2001). In regards to pectin and MCP, in its native form Citrus Pectin (CP) has limited solubility in water and is unable to interact with galectin-3, but in its modified form MCP is able to form small linear water-soluble fibers. These fibers act as a ligand for galectin-3 (Baldwin and Shah, 1993; Cohen et al., 1993; Daas et al., 2001; Gunning et al., 2009). MCP interacts and inactivates galectin-3 activity which is required for cancer cell to cell adhesion/aggregation. The possible role of MCP and its interaction with galectin and cancer cell metastasis is presented in Fig. 2. Recently, Chen et al. (2006) have shown that when LPS-activated macrophages are 
incubated with $90 \%$ esterified pectin they inhibit the activation and binding of $\mathrm{Nf}-\mathrm{kB}$ and $\mathrm{AP}-1$ transcriptional factors to the respective promoter nucleotide sequences and down-regulates the mRNA and protein expression of iNOS and COX2. The activation of transcriptional factors Nf-kB and AP-1 are known to be involved in the pathogenesis of numerous cancers (Fujioka et al., 2004). It is also known that overxpression of iNOS and COX2 by $\mathrm{AP}-1$ and $\mathrm{Nf}-\mathrm{kB}$, respectively, is involved in the pathogenesis of colon cancer (Watanabe et al., 2000). These data clearly suggest that pectin inactivates several key oncogenic factors in cancer as shown in Fig. 3. In another study, Hsieh and Wu (1995) demonstrated that treatment of MCP in human prostate JCA-1 cells downregulates cell cycle regulator cyclin $\mathrm{B}$ and cdc2 proteins resulting in cell arrest in $\mathrm{G} 2 / \mathrm{M}$ phase causing these cells to undergo apoptosis. Inhibition of galectin-3 by pectin polysaccharide or MCP not only possesses antimetastatic properties, but also inhibits cancer cell invasion in MDA-MB-231 human metastatic breast cancer cells and human buccal metastatic cells (Sathisha et al., 2007) and angiogenesis in endothelial cells (Nangia-Makker et al., 2000). These studies reveal that pectin polysaccharides (CP and MCP) inactivate key transcriptional factors, cell cycle regulators and cancer metastatic factor galectin-3, which leads to the inhibition of cancer cell growth, angiogenesis and metastasis.

\subsection{MCP and Chemotherapy}

Several investigations suggest that targeting galectin-3 increases cancer cell sensitivity towards various chemotherapeutic agents such as cisplatin, staurosporine (Yu et al., 2002), etoposide (Fukumori et al., 2006) and doxorubicin (Johnson et al., 2007). From these studies it is clear that pectin, $\mathrm{CP}$ and MCP, as a galectin-3 inhibitor, may hold a significant role in cancer chemotherapy. Interestingly, it has been demonstrated that inhibition of galectin-3 by MCP reverses multiple myeloma cell resistance to bortezomib and enhances apoptosis induced by dexamethasone (Chauhan et al., 2005). Similarly, dose dependent exposure of hemangiosarcoma cells to MCP increases their sensitivity towards doxorubicin and induces apoptosis (Johnson et al., 2007) suggesting that inactivation of galectin-3 by MCP increases sensitivity of cancer cells towards chemotherapeutic agents.

In this scenario, pre-treatment or oral administration of $\mathrm{CP}$ or MCP before chemotherapy would be a better strategy for cancer treatments. A similar study was conducted by Hayashi et al. (2000) where the data clearly suggests that oral administration of MCP $\left(0.8\right.$ and $\left.1.6 \mathrm{mg} \mathrm{mL}^{-1}\right)$, similar to quercetin (a chemopreventive flavonoid) compound in Balb-c-Mice implanted with human colon-25, tumor reduces tumor size by $50-75 \%$ compared with control mice.

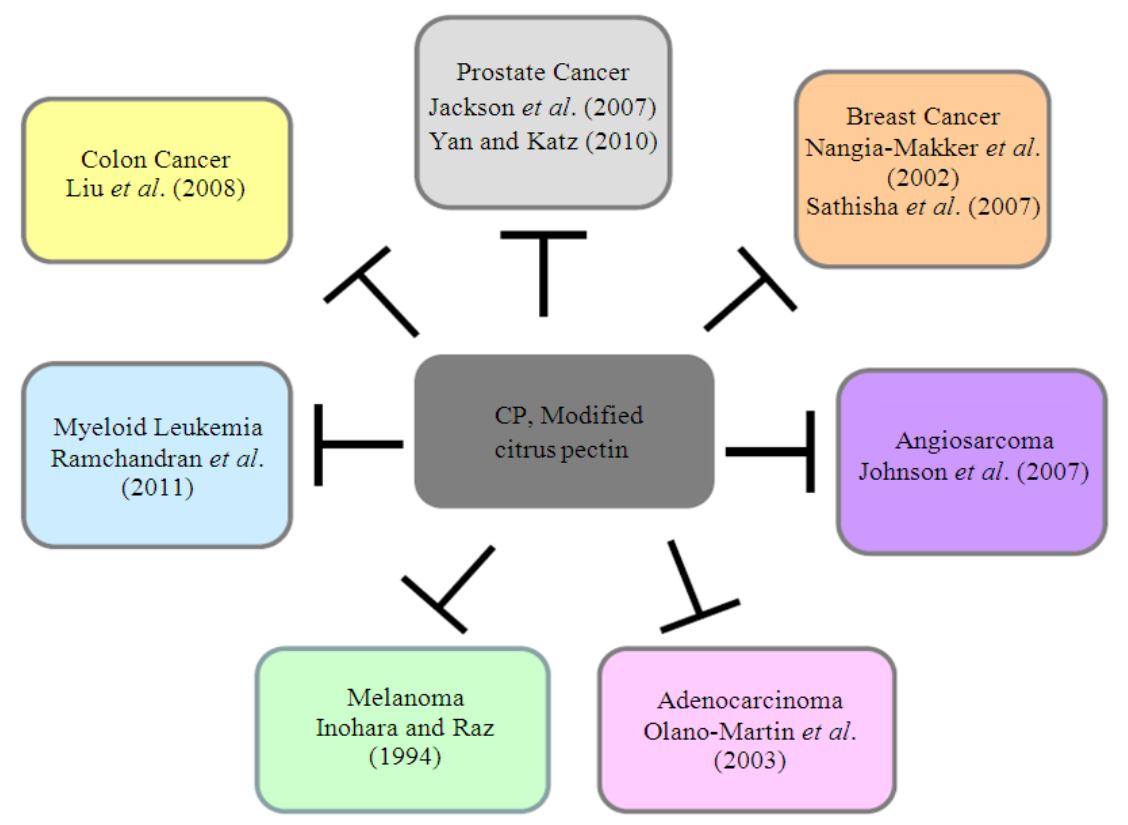

Fig. 4. Possible inhibitory role of $\mathrm{CP}$ and $\mathrm{MCP}$ in suppression of different cancer is presented 
Similarly, oral administration of MCP in mice, which are orthotopically injected with human breast carcinoma MDAMB-435 cells, shows inhibition of tumor growth, angiogenesis and metastasis (Nangia-Makker et al., 2002). These studies indicate that during chemotherapy pre or post administration of MCP could be beneficial for cancer patients. On the other hand, scientists have developed calcium-pectinate beads, which can be used to deliver chemo-preventive drugs in animals. For example, Das and $\mathrm{Ng}$ (2010) have developed resveratrol (an anti-colorectal cancer compound) coupled calcium-pectinate beads. These beads are used to delay the site specific release of resveratrol in the lower GI track. Therefore, utilization of pectic substance, including $\mathrm{CP}$ and $\mathrm{MCP}$, would be beneficial during cancer chemotherapy.

\section{CONCLUSION}

The utilization of natural pectic substances in the medicinal field is always beneficial because of their minimal side effects. In this review we primarily focus on pectin, $\mathrm{CP}, \mathrm{MCP}$ and cancer treatment. A number of studies show that $\mathrm{CP}$ and MCP have potential anticarcinogenic effects and suppress many human cancer cell activities. The literature also suggests that plant pectin, CP and MCP show an immense inhibitory potential against a wide variety of human cancers (Fig. 4). By analyzing the molecular mechanisms, it is now clear that $\mathrm{CP}$ and MCP target and inactivate galectin-3, a cancer cell survival factor, Nf-k $\beta$, a transcription factor involved in tumorigenesis and many kinases and apoptotic markers which leads to suppression of numerous human cancers. Animal studies and human clinical studies show that CP and MCP play a major role in detoxification of metals/carcinogens from the body, which could prevent early stages of human malignancies. Oral injection of pectin, CP and MCP in tumor bearing mice show a significant inhibition of tumor growth, cancer cell metastasis, invasion, angiogenesis and survival. Interestingly, the data also suggests that MCP induces apoptosis in several cancer cells. Future studies should be focused on the isolation of bio-active pectin components which can be useful in cancer drug development.

\section{REFERENCES}

Baldwin, J.L. and A.C. Shah, 1993. Pectin-induced occupational asthma. Chest, 104: 1936-1937. DOI: 10.1378/chest.104.6.1936b

Boyd, D.R. and S.J. Genuis, 2008. The environmental burden of disease in Canada: Respiratory disease, cardiovascular disease, cancer and congenital affliction. Environ. Res., 106: 240-249. DOI: 10.1016/j.envres.2007.08.009
Bresalier, R.S., P.S. Yan, J.C. Byrd, R. Lotan and A. Raz, 1997. Expression of the endogenous galactosebinding protein galectin-3 correlates with the malignant potential of tumors in the central nervous system. Cancer, 80: 776-787. DOI: $10.1002 /($ SICI) 1097 -

0142(19970815)80:4<776::AID-

CNCR17>3.0.CO;2-Q

Brunekreef, B. and S.T. Holgate, 2002. Air pollution and health. Lancet, 360: 1233-1242. DOI: 10.1016/S0140-6736(02)11274-8

Chauhan, D., G. Li, K. Podar, T. Hideshima and P. Neri et al., 2005. A novel carbohydratebased therapeutic GCS-100 overcomes bortezomib resistance and enhances dexamethasone-induced apoptosis in multiple myeloma cells. Cancer Res., 65: 83508358. DOI: 10.1158/0008-5472.CAN-05-0163

Chen, C.H., M.T. Sheu, T.F. Chen, Y.C. Wang and W.C. Hou et al., 2006. Suppression of endotoxin-induced proinflammatory responses by citrus pectin through blocking LPS signaling pathways. Biochem. Pharmacol., 72: 1001-1009. DOI: 10.1016/j.bcp.2006.07.001

Cheng, H., S. Li, Y. Fan, X. Gao and M. Hao et al., 2011. Comparative studies of the antiproliferative effects of ginseng polysaccharides on HT-29 human colon cancer cells. Med. Oncol., 28: 175-181. DOI: 10.1007/s12032-010-9449-8

Cohen, A.J., M.S. Forse and S.M. Tarlo, 1993. Occupational asthma caused by pectin inhalation during the manufacture of jam. Chest, 103: 309-311. DOI: 10.1378/chest.103.1.309

Daas, P.J., B. Boxma, A.M. Hopman, A.G. Voragen and H.A. Schols, 2001. Nonesterified galacturonic acid sequence homology of pectins. Biopolymers, 58: 18. DOI: 10.1002/1097-0282(200101)58:1<1::AIDBIP10>3.0.CO;2-I

Das, S. and K.Y. Ng, 2010. Resveratrol-loaded calciumpectinate beads: Effects of formulation parameters on drug release and bead characteristics. J. Pharm. Sci., 99: 840-860. DOI: 10.1002/jps. 21880

Dumic, J., S. Dabelic and M. Flogel, 2006. Galectin-3: An open-ended story. Biochim. Biophys. Acta., 1760: 616-635. DOI: 10.1016/j.bbagen.2005.12.020

Eliaz, I., A.T. Hotchkiss, M.L. Fishman and D. Rode, 2006. The effect of modified citrus pectin on urinary excretion of toxic elements. Phytother. Res., 20: 859-864. DOI: $10.1002 /$ ptr.1953

Eliaz, I., E. Weil and B. Wilk, 2007. Integrative medicine and the role of modified citrus pectin/aliginates in heavy metal chelation and detoxification-five case reports. Forsch Komplementmed, 14: 358-364. DOI: 10.1159/000109829 
Fisher, H., W.G. Siller and P. Griminger, 1966. The retardation by pectin of cholesterol-induced atherosclerosis in the fowl. J. Atherosclerosis Res., 6: 292-298. DOI: 10.1016/S0368-1319(66)80012-1

Fujioka, S., J. Niu, C. Schmidt, G.M. Sclabas and B. Peng et al., 2004. NF-kappaB and AP-1 connection: Mechanism of NF-kappaB-dependent regulation of AP-1 activity. Mol. Cell. Biol., 24: 7806-7819. PMID: 15314185

Fukumori, T., N. Oka, Y. Takenaka, P. Nangia-Makker and E. Elsamman et al., 2006. Galectin-3 regulates mitochondrial stability and antiapoptotic function in response to anticancer drug in prostate cancer. Cancer Res., 66: 3114-3119. DOI: 10.1158/00085472.CAN-05-3750

Glinskii, O.V., V.H. Huxley, G.V. Glinksy, K.J. Pienta and A. Raz et al., 2005. Mechanical entrapment is insufficient and intercellular adhesion is essential for metastatic cell arrest in distant organs. Neoplasia, 7: 522-527. DOI: 10.1593/neo.04646

Glinsky, V.V. and A. Raz, 2009. Modified citrus pectin anti-metastatic properties: One bullet, multiple targets. Carbohydr. Res., 344: 1788-1791. DOI: 10.1016/j.carres.2008.08.038

Guess, B.W., M.C. Scholz, S.B. Strum, R.Y. Lam and H.J. Johnson et al., 2003. Modified Citrus Pectin (MCP) increases the prostate-specific antigen doubling time in men with prostate cancer: A phase II pilot study. Prostate Cancer Prostatic Dis., 6: 301304. DOI: $10.1038 /$ sj.pcan.4500679

Gunning, A.P., R.J. Bongaerts and V.J. Morris, 2009. Recognition of galactan components of pectin by galectin-3. FASEB J., 23: 415-424. DOI: 10.1096/fj.08-106617

Hayashi, A., A.C. Gillen and J.R. Lott, 2000. Effects of daily oral administration of quercetin chalcone and modified citrus pectin on implanted colon-25 tumor growth in Balb-c mice. Altern. Med. Rev., 5: 546552. PMID: 11134977

Hsieh, T.C. and J.M. Wu, 1995. Changes in cell growth, cyclin/kinase, endogenous phosphoproteins and 23 nm gene expression in human prostatic JCA-1 cells treated with modified citrus pectin. Biochem. Mol. Biol. Int., 37: 833-841. PMID: 8624488

Inohara, H and A. Raz, 1994. Effects of natural complex carbohydrate (citrus pectin) on murine melanoma cell properties related to galectin-3 functions. Glycoconj J., 11: 527-532. DOI: 10.1007/BF00731303

Jackson, C.L., T.M. Dreaden, L.K. Theobald, N.M. Tran and T.L. Beal et al., 2007. Pectin induces apoptosis in human prostate cancer cells: Correlation of apoptotic function with pectin structure. Glycobiology, 17: 805-819. DOI: 10.1093/glycob/cwm054
Jackson, S.P. and J. Bartek, 2009. The DNA-damage response in human biology and disease. Nature, 461: 1071-1078. DOI: $10.1038 /$ nature 08467

Jemal, A., R. Siegel, J. Xu and E. Ward, 2010. Cancer statistics. CA Cancer J. Clin., 60: 277-300. DOI: 10.3322/caac. 20073

Jenkins, D.J, A.R. Leeds, M.A. Gassal, B. Cochet and G.M. Alberti, 1977. Decrease in postprandial insulin and glucose concentrations by guar and pectin. Ann. Intern. Med., 86: 20-23. PMID: 835924

Jenkins, D.J., A.C. Newton, A.R. Leeds and J.H. Cummings, 1975. Effect of pectin, guar gum and wheat fiber on serum cholesterol. Lancet, 1: 116117. PMID: 49474

Jew, S., S.S. AbuMweis and P.J.H. Jones, 2009. Evolution of the human diet: Linking our ancestral diet to modern functional foods as a means of chronic disease prevention. J. Med. Food, 12: 925 934. DOI: $10.1089 /$ jmf.2008.0268

Johnson, K.D., O.V. Glinskii, V.V. Mossine, J.R. Turk and T.P. Mawhinney et al., 2007. Galectin-3 as a potential therapeutic target in tumors arising from malignant endothelia. Neoplasia, 9: 662-670. DOI: 10.1593/neo.07433

Joye, D.D. and G.A. Luzio, 2000. Process for selective extraction of pectins from plant material by differential $\mathrm{pH}$. Carbohydr. Polym., 43: 337-342. DOI: $10.1016 / \mathrm{S} 0144-8617(00) 00191-0$

Kiyohara, H., M. Hirano, X.G. Wen, T. Matsumoto and X.B. Sun et al., 1994. Characterization of an antiulcer pectic polysaccharide from leaves of Panax ginseng C.A. Meyer. Carbohydr. Res., 263: 89-101. DOI: 10.1016/0008-6215(94)00151-0

Krall, S.M. and R.F. Mcfeeters, 1998. Pectin hydrolysis: Effect of temperature, degree of methylation, $\mathrm{pH}$ and calcium on hydrolysis rates. J. Agric. Food Chem., 46: 1311-1315. DOI: 10.1021/jf970473y

Krzeslak, A. and A. Lipinska, 2004. Galectin-3 as a multifunctional protein. Cell. Mol. Biol. Lett., 9: 305-328. PMID: 15213811

Kushi, L.H., T. Byers, C. Doyle, E.V. Bandera and M. McCullough et al., 2006. American cancer society guidelines on nutrition and physical activity for cancer prevention: reducing the risk of cancer with healthy food choices and physical activity. CA Cancer J. Clin., 56: 254-281. DOI: 10.3322/canjclin.56.5.254

Landrigan, P.J., C.B. Schechter, J.M. Lipton, M.C. Fahs and J. Schwartz, 2002. Environmental pollutants and disease in American children: Estimates of morbidity, mortality and costs for lead poisoning, asthma, cancer and developmental disabilities. Environ. Health Perspec., 110: 721-728. DOI: 10.1289/ehp.02110721 
Liu, H.Y., Z.L. Huang, G.H. Yang, W.Q. Lu and N.R. $\mathrm{Yu}, 2008$. Inhibitory effect of modified citrus pectin on liver metastases in a mouse colon cancer model. World J. Gastroenterol., 14: 7386-7391. DOI: 10.3748/wjg. 14.7386

May, C.D., 1990. Industrial pectins: Sources, production and applications. Carbohydr. Polym., 12: 79-99. DOI: 10.1016/0144-8617(90)90105-2

Nangia-Makker, P., V. Hogan, Y. Honjo, S. Baccarini and L. Tait et al., 2002. Inhibition of human cancer cell growth and metastasis in nude mice by oral intake of modified citrus pectin. J. Natl. Cancer Inst., 94: 1854-1862. DOI: 10.1093/jnci/94.24.1854

Nicholas, J., 2009. Fighting cancer metastasis and heavy metal toxicities with modified citrus pectin. Life Extens. Mag.

Niture, S.K., 2008. Comparative biochemical and structural characterizations of fungal polygalacturonases. Biol.: Cellu. Mol. Biol., 63: 119. DOI: $10.2478 / \mathrm{s} 11756-008-0018-y$

Olano-Martin, E., G.H. Rimbach, G.R. Gibson and R.A. Rastall, 2003. Pectin and pecticoligosaccharides induce apoptosis in vitro human colonic adenocarcinoma cells. Anticancer Res., 23: 341-346. PMID: 12680234

Olano-Martin, E., G.R. Gibson and R.A. Rastell, 2002. Comparison of the in vitro bifidogenic properties of pectins and pectic-oligosaccharides. J. Applied Microbiol., 93: 505-511. DOI: 10.1046/j.13652672.2002.01719.x

Perez-Padilla, R., A. Schilmann and H. RiojasRodriguez, 2010. Respiratory health effects of indoor air pollution. Int. J. Tuberc. Lung Dis., 14: 1079-1086. PMID: 20819250

Pienta, K.J., H. Naik, A. Akhtar, K. Yamazaki and T.S. Replogle et al., 1995. Inhibition of spontaneous metastasis in a rat prostate cancer model by oral administration of modified citrus pectin. J. Natl. Cancer Inst., 87: 348-353. DOI: 10.1093/jnci/87.5.348

Platt, D. and A. Raz, 1992. Modulation of the lung colonization of B16-F1 melanoma cells by citrus pectin. J. Natl. Cancer Inst., 84: 438-442. DOI: 10.1093/jnci/84.6.438

Rabbani, G.H., T. Teka, B. Zaman, N. Majid and M. Khatun et al., 2001. Clinical studies in persistent diarrhea: Dietary management with green banana or pectin in Bangladeshi children. Gastroenterology, 121: 554-560. DOI: 10.1053/gast.2001.27178
Ramachandran, C., B.J. Wilk, A. Hotchkiss, H. Chau and I. Eliaz et al., 2011. Activation of human Thelper/inducer cell, T-cytotoxic cell, B-cell and Natural Killer (NK)-Cells and induction of Natural killer cell activity against K562 chronic myeloid leukemia cells with modified citrus pectin. BMC Complement Altern. Med., 11: 59-59. DOI: 10.1186/1472-6882-11-59

Ridley, B.L., M.A. O’Neill and D. Mohnen, 2001. Pectins: Structure, biosynthesis and oligogalacturonide-related signaling. Phytochemistry, 57: 929-967. DOI: 10.1016/S00319422(01)00113-3

Rinaldo, M., 1996. Physicochemical properties of pectins in solution and in gel sates. Progress Biotechnol., 14: 21-33. DOI: 10.1016/S0921-0423(96)80243-7

Sankpal, U., H. Pius, M. Khan, M. Shukoor and P. Maliakal et al., 2012. Environmental factors in causing human cancers: Emphasis on tumorigenesis. Tumor Biol., 33: 1265-1274. DOI: 10.1007/s13277012-0413-4

Sathisha, U.V., S. Jayaram, M.A.H. Nayaka and S.M. Dharmesh, 2007. Inhibition of galectin-3 mediated cellular interactions by pectic polysaccharides from dietary sources. Glycoconj J., 24: 497-507. DOI: 10.1007/s10719-007-9042-3

Srivastava, P. and R. Malviya, 2011. Sources of pectin, extraction and its applications in pharmaceutical industry-An overview. Indian J. Natural Product Resources, 2: 10-18.

Triplehorn, C. and P.S. Millard, 2002. A rice-based diet with green banana or pectin reduced diarrhea in infants better than a rice-alone diet. ACP J. Club., 136: 67-67. PMID: 11874292

Veldman, F.J., C.H. Nair, H.H. Vorster, W.J. Vermaak and J.C. Jerling et al., 1999. Possible mechanisms through which dietary pectin influences fibrin network architecture in hypercholesterolaemic subjects. Thromb. Res., 93: 253-264. DOI: 10.1016/S0049-3848(98)00170-4

Vincken, J.P., H.A. Schols, R.J. Oomen, M.C. McCann and P. Ulvskov et al., 2003. If homogalacturonan were a side chain of rhamnogalacturonan I. Implications for cell wall architecture. Plant Physiol., 132: 1781-1789. DOI: 10.1104/pp.103.022350

Voragen, A.G.J., W. Pilnik, J.F. Thibault, M.A.V. Axelos and C.M.G.C. Renard, 2010. Pectins. In: Food Polysaccharides and their Applications, Stephen A.M. and G.O. Phillips (Eds.), Taylor and Francis, Boca Raton, FL., ISBN-10: 0824759222, pp: 287-339. 
Watanabe, K., T. Kawamori, S. Nakatsugi and K. Wakabayashi, 2000. COX-2 and iNOS, good targets for chemoprevention of colon cancer. Biofactors, 12: 129-133. PMID: 11216473

Willats, W.G., L. McCartney, W. Mackie and J.P. Knox, 2001. Pectin: Cell biology and prospects for functional analysis. Plant Mol. Biol., 47: 9-27. DOI: 10.1023/A:1010662911148

Wong, C.K., K.N. Leung and K.P. Fung, 1994. Immunomodulatory and anti-tumor polysaccharides from medicinal plants. J. Int. Med. Res., 22: 299312. PMID: 7895893

Yan, J. and A.E. Katz, 2010. PectaSol-C modified citrus pectin induces apoptosis and inhibition of proliferation in human and mouse androgendependent and-independent prostate cancer cells. Integr. Cancer Ther., 9: 197-203. DOI: $10.1177 / 1534735410369672$
Yu, F., R.L. Finley, A. Raz and H.R. Kim, 2002. Galectin-3 translocates to the perinuclear membranes and inhibits cytochrome c release from the mitochondria. A role for synexin in galectin-3 translocation. J. Biol. Chem., 277: 15819-15827. DOI: 10.1074/jbc.M200154200

Zhao, Z.Y., L. Liang, X. Fan, Z. Yu and A.T. Hotchkiss et al., 2008. The role of modified citrus pectin as an effective chelator of lead in children hospitalized with toxic lead levels. Altern. Ther. Health Med., 14: 34-38. PMID: 18616067 